\title{
Demographic, Clinical and Management Characteristics of Newly Diagnosed COPD Patients in Turkey: A Real-Life Study
}

This article was published in the following Dove Press journal: International Journal of Chronic Obstructive Pulmonary Disease

\author{
Mecit Suerdem, (D) \\ Hakan Gunen, ${ }^{2}$ \\ Levent Akyildiz, ${ }^{3}$ \\ Aykut Cilli, ${ }^{4}$ Tevfik Ozlu, (1D ${ }^{5}$ \\ Esra Uzaslan, ${ }^{6}$ \\ Oznur Abadoglu, ${ }^{7}$ \\ Hasan Bayram, $\mathbb{D}^{8}$ \\ Arif Hikmet Cimrin, ${ }^{9}$ \\ Bilun Gemicioglu, (D) ${ }^{10}$ \\ Zeynep Misirligil $^{1}$ \\ 'Department of Chest Diseases, Faculty of \\ Medicine, Selcuk University, Konya, Turkey; \\ ${ }^{2}$ Sureyyapasa Chest Diseases and Thoracic \\ Surgery Training and Research Hospital, \\ Health Sciences University, Istanbul, Turkey; \\ ${ }^{3}$ Department of Pulmonary Medicine, \\ Memorial Dicle Hospital, Diyarbakır, Turkey; \\ ${ }^{4}$ Department of Chest Diseases, Faculty of \\ Medicine, Akdeniz University, Antalya, \\ Turkey; ${ }^{5}$ Department of Chest Diseases, \\ Faculty of Medicine, Karadeniz Technical \\ University, Trabzon, Turkey; ${ }^{6}$ Department of \\ Chest Diseases, Faculty of Medicine, Uludag \\ University, Bursa, Turkey; ${ }^{7}$ Private Office of \\ Pulmonary and Allergic Diseases, Istanbul, \\ Turkey; ${ }^{8}$ Koc University Research Center for \\ Translational Medicine (KUTTAM), \\ Department of Pulmonary Medicine, Koc \\ University School of Medicine, Istanbul, \\ Turkey; ${ }^{9}$ Department of Chest Diseases, \\ Faculty of Medicine, Dokuz Eylul University, \\ Izmir, Turkey; ${ }^{10}$ Department of Pulmonary \\ Diseases, Faculty of Cerrahpasa Medicine, \\ Istanbul University-Cerrahpasa, Istanbul, \\ Turkey; "'Department of Pulmonary and \\ Allergic Diseases, Ankara Liv Hospital, \\ Ankara, Turkey
}

Correspondence: Mecit Suerdem Department of Chest Diseases, Faculty of Medicine, Selcuk University, Konya, Turkey

Tel $+903322412181-82$

Fax +903322412184

Email mecsuerdem@gmail.com
Purpose: In order to determine the clinical and sociodemographic characteristics of newly diagnosed treatment-naïve asthma and COPD patients in Turkey, a multicenter study in 2012 was initiated. We aimed to investigate the characteristics and therapies of COPD patients in the original study in more detail.

Patients and Methods: This nation-wide, multicentric, non-interventional, prospective, reallife observational cohort study was conducted in 122 centers. The newly diagnosed patients were not receiving any treatment before the recruitment. Their general characteristics, the combined GOLD 2011 COPD categories and exacerbation histories were noted. The patients were followed up with 3 voluntary visits for 1 year. Their adherence to the inhaled treatment according to GOLD 2011 was evaluated during follow-up visits.

Results: The study included 776 COPD patients. Their mean age was $59.4 \pm 9.1$ years, and $11.9 \%$ of the patients were female. $35.1 \%$ of the patients were in the GOLD $2011 \mathrm{C}$ and D category. $12.6 \%$ are frequent exacerbators, and $52.8 \%$ had at least one comorbid condition. $71.8 \%$ overtreatment rate was detected. Their attendance rates for three follow-up visits became 55.9\%, 32.9\% and $18.7 \%$, respectively. The adherence rate to the treatment was measured as $81.9 \%$.

Conclusion: Although these patients were diagnosed for the first time, the GOLD $\mathrm{C}$ and $\mathrm{D}$ categories and frequent exacerbator phenotype were found at a high rate. They were usually prescribed an overtreatment regimen. We think that newly diagnosed COPD patients should be evaluated carefully, and best effort should be made to treat these patients in accordance with the recommendations of the major COPD guidelines. Keywords: COPD, newly diagnosed, treatment naïve, exacerbations

\section{Introduction}

Chronic obstructive pulmonary disease (COPD) is one of the leading causes of mortality and morbidity worldwide. ${ }^{1,2}$ The population-based surveys of chronic obstructive pulmonary disease (COPD) have confirmed that COPD underdiagnosis is universally high and significant airflow obstruction is often present at the first diagnosis. ${ }^{3,4}$ However, the newly diagnosed patients are expected to be in the early stages. The studies for understanding of different characteristics of newly diagnosed COPD patients are almost lacking. The newly diagnosed COPD patients are presumably to be different from usual COPD patients due to the perception of symptoms and adherence to treatment.

Turkey is among the countries with the highest prevalence of COPD. In the BOLD-Adana Turkey study, the prevalence of COPD was found $19.1 \%(28.5 \%$ in 
men and $10.3 \%$ in women) with a fixed ratio (FEV1/FVC $<70 \%$ ) in adults over 40 years of age. ${ }^{5}$ We published the clinical, therapeutic features and treatment adherence of newly diagnosed treatment-naïve Turkish asthma and COPD patients in an observational multi-centric cohort study. ${ }^{6}$ In this study, since the clinical and demographic characteristics of asthma and COPD patients were presented by comparison, the data of COPD patients could not be presented and discussed in detail. In this study, we aimed to analyze the clinical and therapeutic characteristics of COPD patients in this cohort in detail.

\section{Materials and Methods}

\section{Study Design}

This study is a nation-wide, multi-centric, prospective and non-interventional study focusing on the general characteristics, diagnosis and treatment approaches for newly diagnosed patients with COPD under real-life conditions for 1 year. Written informed consent was obtained from all subjects. The protocol, which was in accordance with the Declaration of Helsinki, was approved by the ethics committee of Erciyes University, Faculty of Medicine (February 10, 2012). The study was conducted between June 2012 and March 2014 in 122 centers (university hospital, public hospital and private hospital) in Turkey. All participants in the study were chest disease specialists.

COPD patients were enrolled on the basis of the GOLD (Global Initiative for COPD) 2011. ${ }^{7}$ A standard web-based questionnaire including items related to demographics, clinical, laboratory and treatment parameters was applied to the patients. All procedures were administered by the investigators (pulmonary physicians) at 122 centers and supervised by the authorized Executive Board consisting of 11 academics for this research organization.

\section{Study Population}

To select the study patients and to avoid bias, the physicians screened all patients consecutively and all eligible patients were informed about the study. Outpatient COPD patients meeting the inclusion criteria below were included in the study:

- Age of diagnosis: $\geq 40$ years to $\leq 80$ years.

- Diagnosis of COPD within the previous 3 months or after the initiation visit.

- Diagnosis of COPD was based on a post-bronchodilator forced expiratory volume in $1 \mathrm{~s}\left(\mathrm{FEV}_{1}\right) /$ forced vital capacity (FVC) ratio of $<0.7$, in the absence of a primary diagnosis of bronchiectasis, asthma or any other significant respiratory disease. The patients also had a smoking history of at least 10 packs/year, or occupational exposure to irritant or toxic gases or biomass exposure.

- Patients (or their legal representatives) who accepted to sign the informed consent form.

\section{Procedures and Measures}

All procedures were supervised by the authorized Executive Board of 11 academics for this research organization. A web-based "Case Report Form" was prepared by the Executive Board and contract research organization. The Case Report Form included previous and current medical history, demographics (the region of life spent longest, the region of life spent in last 3 months, height, body weight, body mass index and education status), risk factors (smoking, occupation, and biomass using), spirometry, disease severity (combined GOLD 2011 criteria for COPD), and the treatment prescribed.

At the first visit, the patients were taken medical history and examined. Spirometry tests were performed, chest radiographs were examined, treatments were arranged and the Case Report Form were completed. After the initial visit, all patients were followed-up for 12 months. Intended timing for the visits was $0-1$ month for visit-1, 1-3 months for visit2 and 3-12 months for visit-3; however, the frequency of the visits was not determined strictly, and the physicians were completely free to plan the follow-up procedures. Data collected during the follow-up visits included the information on exacerbations and assessment of adherence to the treatment. Two or more exacerbations within 1 year were considered to be "frequent exacerbation."

The physician evaluated patients' adherence to the medication at each visit using a scale. According to this scale, "high compliance" defines the patients who use medications regularly, "medium compliance" and "low compliance" terms define those who use medications with partial or significant interruptions, respectively. And finally, "noncompliance" defines the patients who did not use medication at all. The assessment for patients' adherence to the medications was completely based upon their statement.

\section{Data Management}

Physicians were blind to patients of other centers. All lower/upper limits regarding the laboratory test results, decimals, birth dates, etc. were identified in the software 
and entries out of limits were not allowed. System retrieved and weekly updated the package information (name, dosage, pharmaceutical form) of all the medications from the list at the website of Turkish Drug and Medical Device Institution (www.iegm.gov.tr) and allowed physicians to choose the correct and valid name of medication.

To improve data quality, the audit visits were performed at 20 centres ( $15 \%$ of all) which included 450 patients $(22 \%$ of all) and data were compared with the source documents. Data were protected at high capacity servers located at data center of Türk Telekom (communication and substructure provider company). System equipments were designed binary so as to provide back-up in case of any breakdown. Security of the system was provided with software like firewall, antivirus, antispam, IPS (Intrusion Prevention System) and VPN (Virtual Private Network). SSL/VPN (Secure Socket Layer/Virtual Private Network) technology was used for encryption of database. To ensure the secure data transfer, a "https://" (Hyper Text Transfer Protocol Secure) link was used.

\section{Statistical Analysis}

Patients' demographics and disease characteristics were presented as mean $\pm \mathrm{SD}$ for continuous variables and described as percentages for categorical variables.

\section{Results}

A total of 776 newly diagnosed treatment-naïve COPD patients from 122 centers were included. $11.9 \%$ of the patients were female. Their mean age was 59.4 \pm 9.1 years, and more than $50 \%$ of the patients were below 60 years. Current smoker rate was $56.3 \%$. Education level of the study group was low, and approximately $80 \%$ of the patients had less than high school education. $23.4 \%$ of the patients were obese (BMI $\geq 30)$. $60 \%$ of the whole COPD group was living in the cities. Table 1 demonstrates detailed data about the demographic and different characteristics of the patients included in the analysis.

Disease severity assessed during the initial visit using the combined GOLD 2011 criteria revealed the rates from A to D categories $25.7 \%, 39.2 \%, 28.5 \%$ and $6.6 \%$, respectively. More than one-third of the patients (35.1\%) were in the GOLD $2011 \mathrm{C}$ and D categories despite their being newly diagnosed and treatment-naïve COPD patients. The detailed past history pointed out that $12.6 \%$ of the patients were frequent exacerbators ( $\geq 2$ exacerbations in the preceding year), and $4.3 \%$ had hospitalization due to an
Table I Demographic and General Characteristics of the Patients

\begin{tabular}{|l|l|}
\hline Parameters & \\
\hline Age (years)(mean \pm SD) & $59.4 \pm 9.1$ \\
Female/Male (\%) & $11.9 / 88.1$ \\
\hline Age groups (years) (\%) & \\
$40-49$ & 15.5 \\
$50-59$ & 36.9 \\
60-69 & 32.3 \\
$\geq 70$ & 15.3 \\
\hline Smoking status (current/ex) (\%) & $56.3 / 38.1$ \\
Biomass exposure (\%) & 7.2 \\
\hline BMI (kg/m2) (\%) & \\
<25 & 44.4 \\
25-29.9 & 32.2 \\
$\geq 30$ & 23.4 \\
\hline Education (\%) & \\
Illiterate & 11.1 \\
Elementary school & 55.5 \\
Secondary school & 11.7 \\
High school & 15.5 \\
University & 6.2 \\
\hline Living area (\%) & 59.3 \\
Urban & 27.0 \\
Rural & 13.7 \\
Mixed & \\
\hline
\end{tabular}

exacerbation. More than half of the patients (52.8\%) had at least one comorbid condition. The most frequent comorbidities were hypertension $(21.1 \%)$, coronary artery disease (13.6\%) and diabetes mellitus (10.1\%) (Figure 1).

Distribution of the preferred inhaled drug regimens according to the GOLD categories is shown in Table 2. Considering the study group as a whole, the most frequently prescribed drug regimen was ICS+LABA+LAMA combination $(54.9 \%)$. The adherence of the physicians to GOLD 2011 medication recommendations is shown in Table 3. Considering the GOLD 2011 treatment strategy criteria, the overall overtreatment rate was determined as $71.8 \%$. Overtreatment rates were $66.1 \%, 78.7 \%, 81.6 \%$ and $10.6 \%$ according to the GOLD 2011 COPD categories A, B, C and $\mathrm{D}$, respectively.

The rates for visits 1, 2 and 3 in the 1-year follow-up were $55.9 \%, 32.9 \%$ and $18.7 \%$, respectively. According to the patients' statements, $81.9 \%$ of the patients had high adherence to the treatment prescribed. The compliance rates were partially stable between visits in the GOLD groups. 


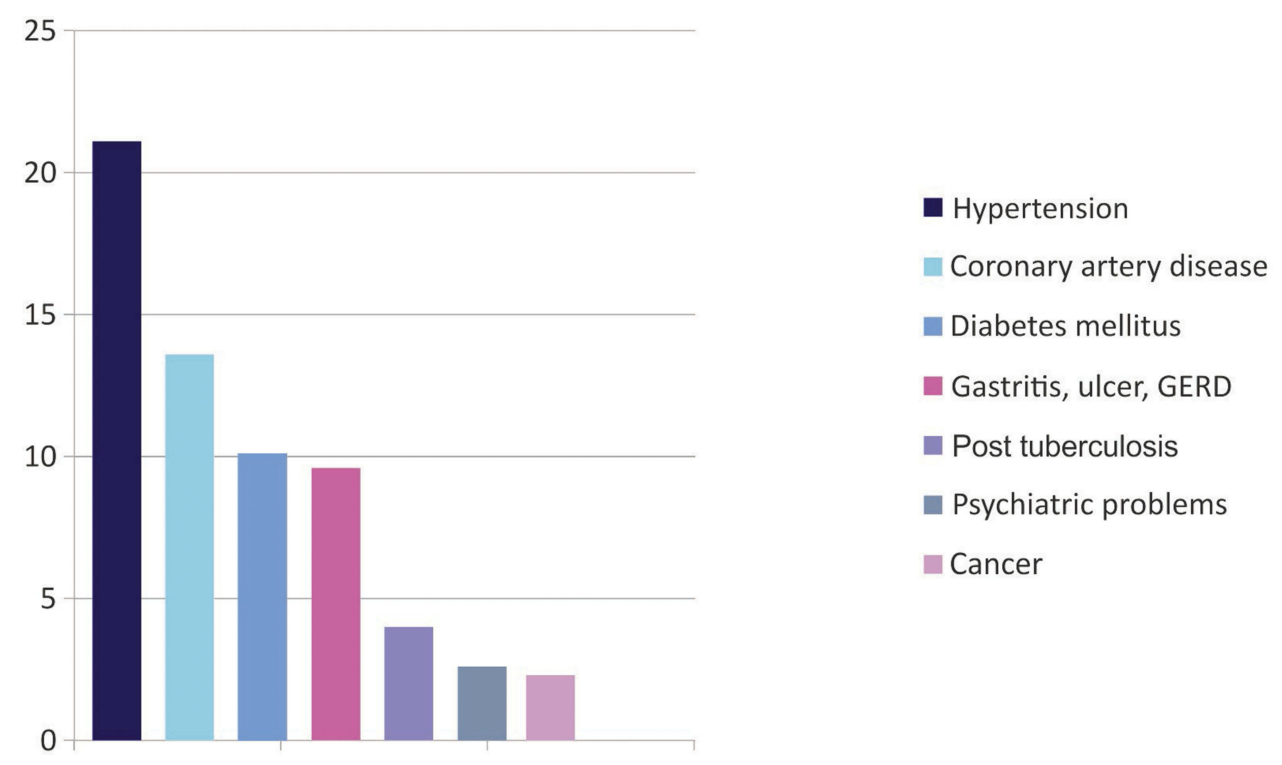

Figure I Frequency of comorbidities in newly diagnosed COPD patients.

\section{Discussion}

This multicentre, non-interventional, prospective, real-life observational study demonstrated the demographical, clinical and management characteristics of the newly diagnosed COPD patients in our country. To our knowledge, this is the first prospective study conducted on newly diagnosed and treatment-naïve COPD patients. Since COPD is closely related to age and is very rare among

Table 2 Distribution of the Preferred Inhaled Drug Regimens According to the GOLD COPD Categories in Newly Diagnosed COPD Patients

\begin{tabular}{|l|l|l|l|l|}
\hline \multirow{2}{*}{ Inhaled Drug Regimens (\%) } & \multicolumn{4}{|l|}{ GOLD Categories } \\
\cline { 2 - 5 } & A & B & C & D \\
\hline ICS/LABA/LAMA & 23.8 & 54.3 & 76.2 & 87.4 \\
ICS/LABA & 27.6 & 23.9 & 18.0 & 10.5 \\
LABA + LAMA & 5.5 & 8.3 & 1.5 & 2.1 \\
LAMA & 24.5 & 10.4 & 1.5 & - \\
LABA & 5.4 & 2.6 & 1.0 & - \\
Others & 13.2 & 0.5 & 1.8 & - \\
\hline
\end{tabular}

Table 3 Adherence to GOLD 20I I Treatment Recommendations of Physicians

\begin{tabular}{|l|l|l|l|l|l|}
\hline \multirow{2}{*}{} & \multirow{2}{*}{ Total } & \multicolumn{4}{|l|}{ GOLD Categories } \\
\cline { 3 - 6 } & & A & B & C & D \\
\hline Appropriate & 27.8 & 33.9 & 20.9 & 17.4 & 89.4 \\
Overtreatment & 71.8 & 66.1 & 78.7 & 81.6 & 10.6 \\
Undertreatment & 0.4 & - & 0.4 & 1.0 & - \\
\hline
\end{tabular}

subjects below 40 years of age and elderly patients have difficulty performing spirometry correctly, ${ }^{8-10}$ patients 40 years and over and 80 years and under were included in the study. The important findings of the study were that approximately one-third of newly diagnosed COPD patients had the GOLD stage C or D disease severity, and more than $70 \%$ of the patients were over treated. The detailed past history pointed out that $12.6 \%$ of our patients were frequent exacerbators before COPD diagnosis. This high exacerbation rate in newly diagnosed COPD patient group is a very important finding because COPD exacerbations are a major cause of morbidity and mortality.

Generally mild to moderate COPD patients are underdiagnosed and the diagnosis of COPD is usually made in moderate or severe stages. ${ }^{11}$ Delay in diagnosis of COPD is a global and multifactorial problem. ${ }^{4}$ In a Spain study, COPD prevalence was found $10 \%$ and only $27 \%$ of these patients had COPD diagnosis before. ${ }^{12}$ In a Norway study, only $20 \%$ of male patients and $30 \%$ of female patients were diagnosed COPD by a physician. ${ }^{8}$ The rate of underdiagnosed COPD patients in Turkey is much higher than European countries. In the BOLD-Adana study, 8.4\% patients had COPD diagnosis. ${ }^{5}$ The most important reason of delay in COPD diagnosis is the slowly progressive nature of COPD. ${ }^{1}$ Therefore, the disease usually remains undetected, and a large number of patients are first determined during the exacerbation period. Another important reasons for the late diagnosis are the lack of awareness about COPD in health care providers, ${ }^{13}$ underuse of 
spirometry in health care centers ${ }^{14,15}$ and low awareness of COPD among the general population. ${ }^{16,17}$

Only $5.6 \%$ of the patients were nonsmokers. $7.2 \%$ of patients had biomass fuels use in their history. In lowincome countries, exposure to biomass fuels for cooking and heating is an important cause of COPD in rural areas of developing and the least developed countries. ${ }^{18,19}$ Biomass smoke is an important contributing factor to the development of chronic airway diseases in non-smoker females in the Central, East, and South Eastern Anatolian Regions of Turkey. ${ }^{20,21}$ Underdiagnosis of COPD was frequent in women with exposure biomass fuels due to a lack of knowledge of hazards of biomass smoke, the low level of socioeconomic status and the ignorance of the health care providers. ${ }^{22}$

In the same period with this study, another study called the ALPHABET with a similar methodology was conducted on usual COPD outpatients (n:1610). ${ }^{23}$ In both studies, the demographic characteristics of the patients were similar. However, the mean age of our study groups was found approximately 3 years younger than the usual COPD outpatients. Our findings have shown that $35.1 \%$ of the patients were labeled as GOLD C and D category. Interestingly, the ALPHABET study also yielded that $38.2 \%$ of the usual outpatients were identified as in the GOLD C and D categories. ${ }^{23}$ These figures from newly diagnosed treatment-naïve outpatients and usual outpatients were quite similar.

COPD patients have a large number of comorbidities, and these comorbidities can also be seen in newly diagnosed patients. ${ }^{24}$ The comorbidities of COPD may increase the risk of morbidity and mortality. ${ }^{25}$ We found that at least one comorbidity was present in $52.8 \%$ of the patients. Although the comorbidity rate in our study was found comparably less than the previous studies (65-97\%), their rank order was almost the same. ${ }^{26,27}$

Our analysis demonstrated that the majority of COPD patients with the GOLD stage A, B and C disease severity are overtreated. Although the patients in the present study were newly diagnosed and treatment-naïve ones, the overtreatment rate was found as $71.8 \%$. The most frequently prescribed medication was triple regimen (ICS+LABA +LAMA) with an overall frequency of $54.9 \%$. The current studies show that very high over-treatment rates in COPD are a major global problem. ${ }^{28}$ In a study conducted in 2010 in Turkey, overtreatment was determined in $100 \%$ of the GOLD stage 1 and $91.1 \%$ of the GOLD stage 2 contrary to GOLD recommendations. ${ }^{29}$ In the ALPHABET study, over-treatment was also noted in $>70 \%$ of GOLD 2013 $\mathrm{A}, \mathrm{B}$, and $\mathrm{C}$ patients. ${ }^{23}$ Compliance with COPD guidelines has been shown to be globally low in several studies, and adherence to the GOLD treatment strategy is not sufficient. ${ }^{30-32}$ Other reasons for nonadherence in treatment may be the inability of physicians to perceive the severity of illness and the maintenance of ICS use habits.

The patients' adherence to the visits during 1-year of follow-up is poor in our population. We planned 3 voluntary follow-up visits for our patients during 1-year after the initial visit. Considering the 1-year follow-up visits, the attendance rates of our patient groups were moderate, $55.9 \%, 32.9 \%$ and $18.7 \%$, respectively. Based upon the patients' statements, the adherence rate to the prescribed inhaled regimens was reportedly very high (81.9\%). Adherence rates in COPD are known to vary from $22 \%$ to $78 \%{ }^{33-35}$ Good adherence is associated with reduced exacerbation rates in patients with COPD. ${ }^{36} \mathrm{~A}$ subgroup analysis of the TORCH (Towards a Revolution in COPD Health) study showed that good compliance to study medication in patients with COPD was associated with lower mortality rates compared with poor compliance $(11.3 \%$ vs 26.4\%). ${ }^{37}$ Our study showed that adherence rates to the treatment decrease as the category becomes more severe from the GOLD stage A to D. This general trend is reasonable since less severe patients get more benefit from the prescribed therapy, and thus become comparably more adherent to the treatment.

In this study, $56 \%$ of COPD patients were active smokers. In a study to identify subgroups of COPD using cluster analysis in ECLIPSE (Evaluation of COPD Longitudinally to Identify Predictive Surrogate Endpoints), the rate of active smokers was between $25 \%$ and $42 \%$ (mean 36\%). ${ }^{38}$ In a previous study by Gunen et al, the smoking rate in COPD was found to be unexpectedly high in the Malatya region of Turkey. ${ }^{20}$ In this study, $25.5 \%$ of the women and $57.2 \%$ of the men in the general population were current smokers. The prevalence of COPD was $18.1 \%$ in current smokers over 40 years of age, and $77.5 \%$ of the participants with COPD were current smokers.

This study has several limitations. The major limitation of the study is that the follow-up of patients was not regular due to the study design. Second, the determination of treatment adherence during a 1-year follow-up has some limitations. Selection bias could be a problem because surprisingly high adherence rates were obtained from the patients who attended the follow-up visits. Additionally, since the determination of treatment adherence was 
completely based upon the patients' statements, some misleading statements might have increased our adherence rates significantly. On the other hand, we believe that the general trends of treatment adherence revealed and the distributions according to the GOLD categories were less affected by this relatively subjective approach.

\section{Conclusion}

To our knowledge, this is the first prospective real-life study on newly diagnosed and treatment-naïve COPD patients. Although these patients were diagnosed for the first time, the GOLD C and D categories and frequent exacerbator phenotype were found at a high rate. Our data have shown that although this subgroup of patients is younger than the usual COPD patients with fully established disease, their comorbidity rates are high and $35 \%$ of these patients have a high risk for exacerbations (the GOLD categories $\mathrm{C}$ and $\mathrm{D}$ ). They were usually prescribed an overtreatment regimen. In conclusion, every tool should be used to increase the awareness of COPD and the effective early detection program for COPD in our community. The newly diagnosed, treatment-naïve COPD patients should be evaluated carefully, and best effort should be made to treat these patients in accordance with the recommendations of the major COPD guidelines.

\section{Disclosure}

The study is financially supported by "Abdi Ibrahim Ilac San. ve Tic. AS, Turkey." All authors are members of the advisory board of the study and received payment from "Abdi Ibrahim Ilac San. ve Tic. AS, Turkey" for this. Professor Hakan Gunen reports non-financial support from AbdiIbrahim Pharmaceutical Company, during the conduct of the study; Abdiİbrahim Turkey Pharmaceutical Company paid the publication processing fee, outside the submitted work. Dr Tevfik Ozlu reports personal fees from Abdiibrahim, during the conduct of the study; Prof. Dr. Oznur Abadoglu reports grants from Abdi İbrahim, during the conduct of the study. Dr Bilun Gemicioglu reports Clinical research grants from Sanofi, AstraZeneca, Novartis; Consultancy or lecture grants from Abdi Ibrahim, Deva, Novartis, during the conduct of the study. Prof. Dr. Zeynep Misirligil report grants, personal fees from Abdi İbrahim İlaç San. ve Tic. AS, during the conduct of the study. The authors report no other conflicts of interest in this work.

\section{References}

1. Global Strategy for the Diagnosis, Management and Prevention of COPD, Global Initiative for Chronic Obstructive Lung Disease (GOLD). 2019. Available from: http://www.goldcopd.org/. Accessed December 29, 2018.

2. Buist AS, Vollmer WM, McBurnie MA. Worldwide burden of COPD in high- and low-income countries. Part I. The Burden of Obstructive Lung Disease (BOLD) Initiative. Int J Tuberc Lung Dis. 2008;12 (7):703-708.

3. Celli BR, MacNee W. Standards for the diagnosis and treatment of patients with COPD: a summary of the ATS/ERS position paper. Eur Respir J. 2004;23(6):932-946. doi:10.1183/09031936.04.00014304

4. Lamprecht B, Soriano JB, Studnicka M, et al. Determinants of underdiagnosis of COPD in national and international surveys. Chest. 2015;148(4):971-985. doi:10.1378/chest.14-2535

5. Kocabas A, Hancioglu A, Turkyilmaz S, et al. Prevalence of COPD in Adana, Turkey (BOLD-Turkey Study). Proc Am Thorac Soc. 2006;3 (suppl):A543.

6. Misirligil Z, Cimrin A, Gunen H, et al. Real life profile of asthma and chronic obstructive pulmonary disease patients in Turkey. Tuberk Toraks. 2017;65:169-179. doi:10.5578/tt.54065

7. Global Strategy for the Diagnosis, Management and Prevention of COPD, Global Initiative for Chronic Obstructive Lung Disease (GOLD). 2011.

8. Waatevik M, Skorge TD, Omenaas E, Bakke PS, Gulsvik A, Johannessen A. Increased prevalence of chronic obstructive pulmonary disease in a general population. Respir Med. 2013;107:1037-1045. doi:10.1016/j.rmed.2013.04.008

9. Allen SC, Baxter M. A comparison of four tests of cognition as predictors of inability to perform spirometry in old age. Age Ageing. 2009;38(5):537-541. doi:10.1093/ageing/afp104

10. Carvalhaes-Neto N, Lorino H, Gallinari C, et al. Cognitive function and assessment of lung function in the elderly. Am J Respir Crit Care Med. 1995;152(5):1611-1615. doi:10.1164/ajrccm.152.5.7582303

11. Walters JA, Hansen EC, Walters EH, Wood-Baker R. Underdiagnosis of chronic obstructive pulmonary disease: a qualitative study in primary care. Respir Med. 2008;102(5):738-743. doi:10.1016/j. rmed.2007.12.008

12. Miravitlles M, Soriano JB, Garcia-Rio F, et al. Prevalence of COPD in Spain: impact of underdiagnosis COPD on quality of life and daily life activities. Thorax. 2009;64(10):863-868. doi:10.1136/thx.2009.115725

13. Yawn BP, Wollan PC. Knowledge and attitudes of family physicians coming to COPD continuing medical education. Int J Chron Obstruct Pulmon Dis. 2008;3(2):311-317. doi:10.2147/COPD.S2486

14. Soriano JB, Zielinski J, Price D. Screening for and early detection of chronic obstructive pulmonary disease. Lancet. 2009;374 (9691):721-732. doi:10.1016/S0140-6736(09)61290-3

15. Caramori G, Bettoncelli G, Tosatto R, et al. Underuse of spirometry by general practitioners for the diagnosis of COPD in Italy. Monaldi Arch Chest Dis. 2005;63(1):6-12. doi:10.4081/monaldi.2005.651

16. Miravitlles M, de la Roza C, Morera J, et al. Chronic respiratory symptoms, spirometry and knowledge of COPD among general population. Respir Med. 2006;100(11):1973-1980. doi:10.1016/j. rmed.2006.02.024

17. Halding AG, Heggdal K, Wahl A. Experiences of self-blame and stigmatisation for self-infliction among individuals living with COPD. Scand J Caring Sci. 2011;25(1):100-107. doi:10.1111/j.1471-6712. 2010.00796.x

18. Bruce N, Perez-Padilla R, Albalak R. Indoor air pollution in developing countries: a major environmental and public health challenge. Bull World Health Organ. 2000;78(9):1078-1092.

19. Salvi S, Barnes PJ. Is exposure to biomass smoke the biggest risk factor for COPD globally? Chest. 2010;138(1):3-6. doi:10.1378/ chest.10-0645 
20. Günen H, Hacıevliyagil SS, Yetkin O, et al. Prevalence of COPD: first epidemiological study of a large region in Turkey. Eur J Intern Med. 2008;19(7):499-504. doi:10.1016/j.ejim.2007.06.028

21. Ekici A, Ekici M, Kurtipek E, et al. Obstructive airway diseases in women exposed to biomass smoke. Environ Res. 2005;99(1):93-98. doi:10.1016/j.envres.2005.01.004

22. Mahishale VK, Angadi N, Metgudmath V, et al. The prevalence of chronic obstructive pulmonary disease and the determinants of underdiagnosis in women exposed to biomass fuel in india- a cross section study. Chonnam Med J. 2016;52:117-122. doi:10.4068/cmj.2016.52.2.117

23. Günen H, Yılmaz M, Aktas O, et al. Categorization of COPD patients in Turkey via GOLD 2013 strategy document: ALPHABET study. Int J Chron Obstruct Pulmon Dis. 2015;10:2485-2494. doi:10.2147/ COPD

24. Soriano JB, Visick GT, Muellerova H, et al. Patterns of comorbidities in newly diagnosed COPD and asthma in primary care. Chest. 2005;128(4):2099-2107. doi:10.1378/chest.128.4.2099

25. Sin DD, Anthonisen NR, Soriano JB, Agusti AG. Mortality in COPD: role of comorbidities. Eur Respir J. 2006;28(6):1245-1257. doi:10.1183/09031936.00133805

26. Frei A, Muggensturm P, Putcha N, et al. Five comorbidities reflected the health status in patients with chronic obstructive pulmonary disease: the newly developed COMCOLD index. J Clin Epidemiol. 2014;67(8):904-911. doi:10.1016/j.jclinepi.2014.03.005

27. Miller J, Edwards LD, Agustí A, et al. Comorbidity, systemic inflammation and outcomes in the ECLIPSE cohort. Respir Med. 2013;107 (9):1376-1384. doi:10.1016/j.rmed.2013.05.001

28. Sharif R, Cuevas CR, Wang Y, et al. Guideline adherence in management of stable chronic obstructive pulmonary disease. Respir Med. 2013;107(7):1046-1052. doi:10.1016/j.rmed.2013.04.001

29. Şen E, Güçlü SZ, Kibar I, et al. Adherence to GOLD guideline treatment recommendations among pulmonologists in Turkey. Int J Chron Obstruct Pulmon Dis. 2015;10:2657-2663. doi:10.21 47/COPD.S85324
30. Jochmann A, Neubauer F, Miedinger D, et al. General practitioner's adherence to the COPD GOLD guidelines: baseline data of the Swiss COPD Cohort Study. Swiss Med Wkly. 2010;140:w13053.

31. Seaman J, Leonard AC, Panos RJ. Health care utilization history, GOLD guidelines, and respiratory medication prescriptions in patients with COPD. Int J Chron Obstruct Pulmon Dis. 2010;5:89-97. doi:10.2147/ copd.s8822

32. Fitch K, Iwasaki K, Pyenson B, et al. Variation in adherence with Global Initiative for Chronic Obstructive Lung Disease (GOLD) drug therapy guidelines: a retrospective actuarial claims data analysis. Curr Med Res Opin. 2011;27:1425-1429. doi:10.1185/03007995.2011.583230

33. Makela MJ, Backer V, Hedegaard M, Larsson K. Adherence to inhaled therapies, health outcomes and costs in patients with asthma and COPD. Respir Med. 2013;107(10):1481-1490. doi:10.1016/j. rmed.2013.04.005

34. Krigsman K, Nilsson JL, Ring L. Refill adherence for patients with asthma and COPD: comparison of a pharmacy record database with manually collected repeat prescriptions. Pharmacoepidemiol Drug Saf. 2007;16(4):441-448. doi:10.1002/(ISSN)1099-1557

35. Restrepo RD, Alvarez MT, Wittnebel LD, et al. Medication adherence issues in patients treated for COPD. Int J Chron Obstruct Pulmon Dis. 2008;3(3):371-384. doi:10.2147/COPD.S3036

36. Chrystyn H, Small M, Milligan G, et al. Impact of patients' satisfaction with their inhalers on treatment compliance and health status in COPD. Respir Med. 2014;108(2):358-365. doi:10.1016/j.rmed.2013.09.021

37. Vestbo J, Anderson JA, Calverley PM, et al. Adherence to inhaled therapy, mortality and hospital admission in COPD. Thorax. 2009;64 (11):939-943. doi:10.1136/thx.2009.113662

38. Rennard SI, Locantore N, Delafont B, et al. Identification of five chronic obstructive pulmonary disease subgroups with different prognoses in the ECLIPSE Cohort using cluster analysis. Ann Am Thorac Soc. 2015;12(3):303-312. doi:10.1513/AnnalsATS.201403-125OC

International Journal of Chronic Obstructive Pulmonary Disease

Dovepress

\section{Publish your work in this journal}

The International Journal of COPD is an international, peer-reviewed journal of therapeutics and pharmacology focusing on concise rapid reporting of clinical studies and reviews in COPD. Special focus is given to the pathophysiological processes underlying the disease, intervention programs, patient focused education, and self management protocols. This journal is indexed on PubMed Central, MedLine and CAS. The manuscript management system is completely online and includes a very quick and fair peer-review system, which is all easy to use. Visit http://www.dovepress.com/testimonials.php to read real quotes from published authors. 